\title{
BOOTP Vendor Information Extensions
}

Status of this Memo

This memo is a status report on the vendor information extensions used in the Bootstrap Protocol (BOOTP). Distribution of this memo is unlimited.

Introduction

This RFC is a slight revision and extension of RFC-1048 by Philip Prindeville, who should be credited with the original work in this memo. This memo will be updated as additional tags are are defined. This edition introduces Tag 18 for Extension Path.

As workstations and personal computers proliferate on the Internet, the administrative complexity of maintaining a network is increased by an order of magnitude. The assignment of local network resources to each client represents one such difficulty. In most environments, delegating such responsibility to the user is not plausible and, indeed, the solution is to define the resources in uniform terms, and to automate their assignment.

The basic Bootstrap Protocol [RFC-951] dealt with the issue of assigning an internet address to a client, as well as a few other resources. The protocol included provisions for vendor-defined resource information.

This memo defines a (potentially) vendor-independent interpretation of this resource information.

Overview of BOOTP

While the Reverse Address Resolution (RARP) Protocol [RFC-903] may be used to assign an IP address to a local network hardware address, it provides only part of the functionality needed. Though this protocol can be used in conjunction with other supplemental protocols (the Resource Location Protocol [RFC-887], the Domain Name System [RFC1034]), a more integrated solution may be desirable. 
Bootstrap Protocol (BOOTP) is a UDP/IP-based protocol that allows a booting host to configure itself dynamically, and more significantly, without user supervision. It provides a means to assign a host its IP address, a file from which to download a boot program from some server, that server's address, and (if present) the address of an Internet gateway.

One obvious advantage of this procedure is the centralized management of network addresses, which eliminates the need for per-host unique configuration files. In an environment with several hundred hosts, maintaining local configuration information and operating system versions specific to each host might otherwise become chaotic. By categorizing hosts into classes and maintaining configuration information and boot programs for each class, the complexity of this chore may be reduced in magnitude.

BooTP Vendor Information Format

The full description of the BOOTP request/reply packet format may be found in [RFC-951]. The rest of this document will concern itself with the last field of the packet, a 64 octet area reserved for vendor information, to be used in a hitherto unspecified fashion. A generalized use of this area for giving information useful to a wide class of machines, operating systems, and configurations follows. In situations where a single BOOTP server is to be used among heterogeneous clients in a single site, a generic class of data may be used.

Vendor Information "Magic Cookie"

As suggested in [RFC-951], the first four bytes of this field have been assigned to the magic cookie, which identifies the mode in which the succeeding data is to be interpreted. The value of the magic cookie is the 4 octet dotted decimal 99.130.83.99 (or hexadecimal number 63.82.53.63) in network byte order.

Format of Individual Fields

The vendor information field has been implemented as a free format, with extendable tagged sub-fields. These sub-fields are length tagged (with exceptions; see below), allowing clients not implementing certain types to correctly skip fields they cannot interpret. Lengths are exclusive of the tag and length octets; all multi-byte quantities are in network byte-order. 
Fixed Length Data

The fixed length data are comprised of two formats. Those that have no data consist of a single tag octet and are implicitly of one-octet length, while those that contain data consist of one tag octet, one length octet, and length octets of data.

Pad Field (Tag: 0, Data: None)

May be used to align subsequent fields to word boundaries required by the target machine (i.e., 32-bit quantities such as IP addresses on 32-bit boundaries).

Subnet Mask Field (Tag: 1, Data: 4 subnet mask bytes)

Specifies the net and local subnet mask as per the standard on subnetting [RFC-950]. For convenience, this field must precede the GATEWAY field (below), if present.

Time Offset Field (Tag: 2, Data: 4 time offset bytes) Specifies the time offset of the local subnet in seconds from Coordinated Universal Time (UTC); signed 32-bit integer.

End Field (Tag: 255, Data: None)

Specifies end of usable data in the vendor information area. The rest of this field should be filled with PAD zero) octets.

Variable Length Data

The variable length data has a single format; it consists of one tag octet, one length octet, and length octets of data.

Gateway Field (Tag: 3, Data: N address bytes)

Specifies the IP addresses of N/4 gateways for this subnet. If one of many gateways is preferred, that should be first.

Time Server Field (Tag: 4, Data: N address bytes)

Specifies the IP addresses of N/4 time servers [RFC-868].

IEN-116 Name Server Field (Tag: 5, Data: N address bytes)

Specifies the IP addresses of N/4 name servers [IEN-116]. 


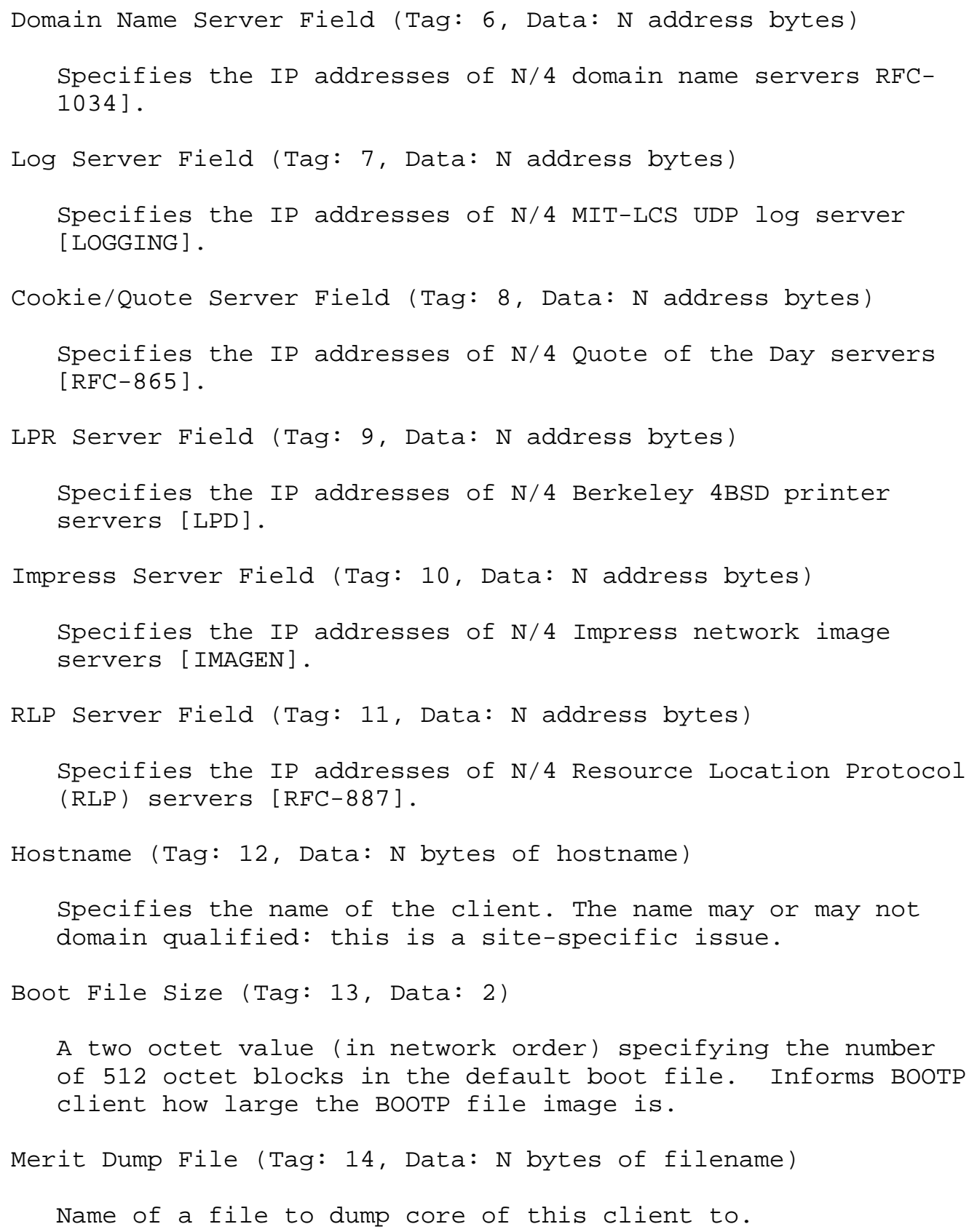




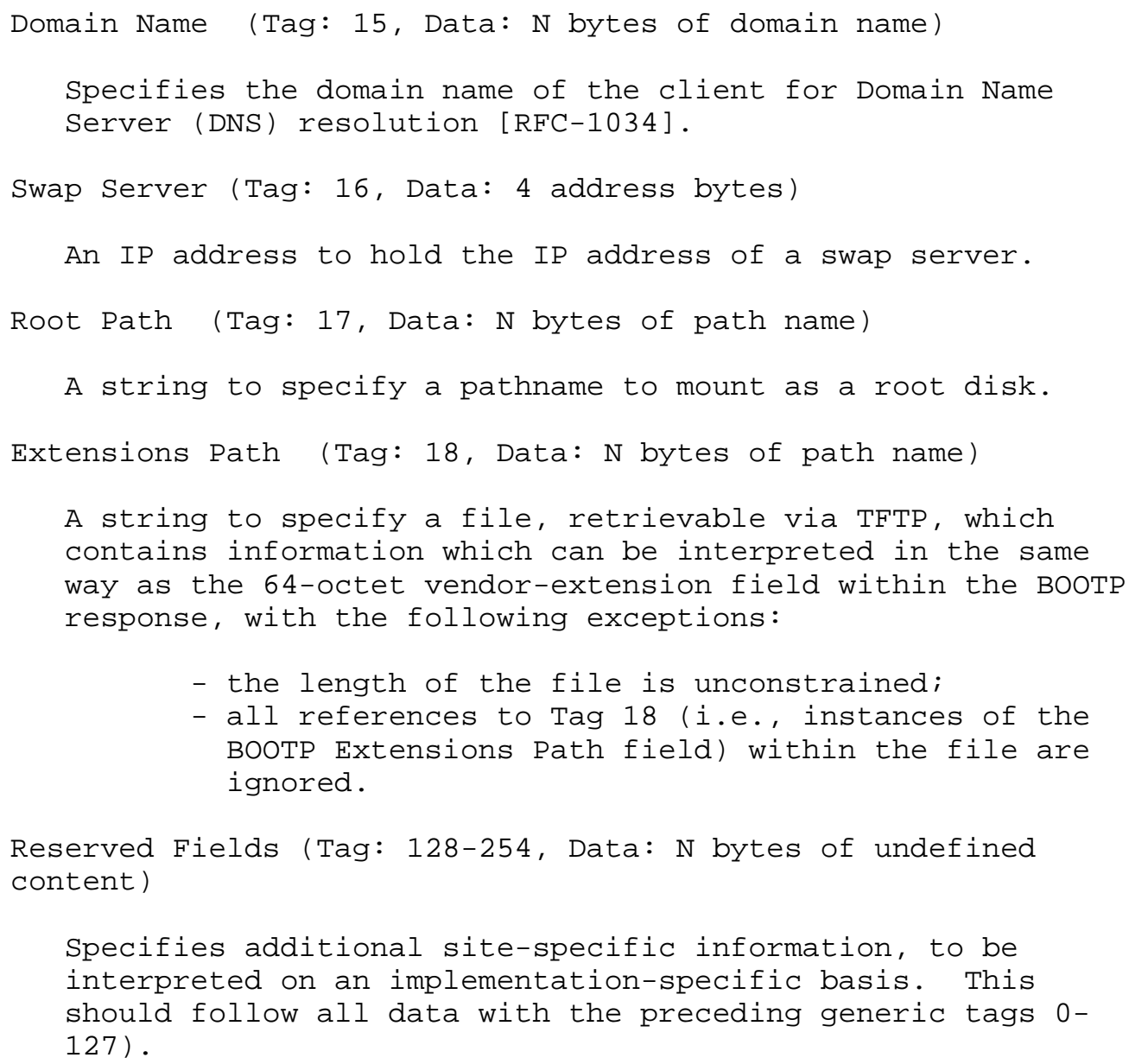

Extensions

Additional generic data fields may be registered by contacting:

Internet Assigned Numbers Authority (IANA)

Information Sciences Institute

University of Southern California

4676 Admiralty Way

Marina del Rey, California 90292-6695

or by email as: ianalisi.edu

Implementation specific use of undefined generic types (those in the range 19-127) may conflict with other implementations, and registration is required. 
When selecting information to put into the vendor specific area, care should be taken to not exceed the 64 byte length restriction. Nonessential information (such as host name and quote of the day server) may be excluded, which may later be located with a more appropriate service protocol, such as RLP or the WKS resource-type of the domain name system. Indeed, even RLP servers may be discovered using a broadcast request to locate a local RLP server.

Comparison to Alternative Approaches

Extending BOOTP to provide more configuration information than the minimum required by boot PROMs may not be necessary. Rather than having each module in a host (e.g., the time module, the print spooler, the domain name resolver) broadcast to the BOOTP server to obtain the addresses of required servers, it would be better for each of them to multicast directly to the particular server group of interest, possibly using "expanding ring" multicasts.

The multicast approach has the following advantages over the BOOTP approach:

- It eliminates dependency on a third party (the BooTP server) that may be temporarily unavailable or whose database may be incorrect or incomplete. Multicasting directly to the desired services will locate those servers that are currently available, and only those.

- It reduces the administrative chore of keeping the (probably replicated) BOOTP database up-to-date and consistent. This is especially important in an environment with a growing number of services and an evolving population of servers.

- In some cases, it reduces the amount of packet traffic and/or the delay required to get the desired information. For example, the current time can be obtained by a single multicast to a time server group which evokes replies from those time servers that are currently up. The BOOTP approach would require a broadcast to the BOOTP server, a reply from the BOOTP server, one or more unicasts to time servers (perhaps waiting for long timeouts if the initially chosen server(s) are down), and finally a reply from a server.

One apparent advantage of the proposed BOOTP extensions is that they provide a uniform way to locate servers. However, the multicast approach could also be implemented in a consistent way across multiple services. The V system naming protocol is a good example of this; character string pathnames are used to name any number of resources (i.e., not just files) and a standard subroutine library looks after multicasting to locate the resources, caching the discovered locations, and detecting stale cache data. 
Another apparent advantage of the BOOTP approach is that it allows an administrator to easily control which hosts use which servers. The multicast approach favors more distributed control over resource allocation, where each server decides which hosts it will serve, using whatever level of authentication is appropriate for the particular service. For example, time servers usually don't care who they serve (i.e., administrative control via the BOOTP database is unnecessary), whereas file servers usually require strong authentication (i.e., administrative control via the BOOTP database is insufficient).

The main drawback of the multicast approach, of course, is that IP multicasting is not widely implemented, and there is a need to locate existing services which do not understand IP multicasts.

The BOOTP approach may be most efficient in the case that all the information needed by the client host is returned by a single BOOTP reply and each program module simply reads the information it needs from a local table filled in by the BooTP reply.

Acknowledgments

The following people provided helpful comments on the first edition of this memo: Drew Perkins, of Carnagie Mellon University, Bill Croft, of Stanford University, and co-author of BOOTP, and Steve Deering, also of Stanford University, for contributing the "Comparison to Alternative Approaches" section.

References

[RFC-951] Croft, B., and J. Gilmore, "Bootstrap Protocol (BOOTP)", RFC 951, Stanford and SUN Microsystems, September 1985.

[RFC-903] Finlayson, R., Mann, T., Mogul, J., and M. Theimer, "A Reverse Address Resolution Protocol", STD 38, RFC 903, Stanford, June 1984.

[RFC-887] Accetta, M., "Resource Location Protocol", RFC 887, CMU, December 1983 .

[RFC-1034] Mockapetris, P., "Domain Names - Concepts and Facilities", STD 13, RFC 1034, USC/Information Sciences Institute, November 1987.

[RFC-950] Mogul, J., and J. Postel, "Internet Standard Subnetting Procedure", STD 5, RFC 950, USC/Information Sciences Institute, August 1985. 
[RFC-868] Postel, J., "Time Protocol", STD 26, RFC 868, USC/Information Sciences Institute, May 1983.

[IEN-116] Postel, J., "Internet Name Server", USC/Information Sciences Institute, August 1979.

[LOGGING] Clark, D., "Logging and Status Protocol", Massachusetts Institute of Technology Laboratory for Computer Science, Cambridge, Massachusetts, 1981.

[RFC-865] Postel, J., "Quote of the Day Protocol", STD 23, RFC 865, USC/Information Sciences Institute, May 1983.

[LPD] Campbell, R., "4.2BSD Line Printer spooler Manual", UNIX Programmer's Manual, Vol II, University of California at Berkeley, Computer Science Division, July 1983.

[IMAGEN] "Image Server XT Programmer's Guide", Imagen Corporation, Santa Clara, California, August 1986.

Security Considerations

Security issues are not discussed in this memo.

Author's Address:

Joyce K. Reynolds

Information Sciences Institute University of Southern California

4676 Admiralty Way

Marina del Rey, CA 90292

Phone: (310) 822-1511

EMail: jkrey@isi.edu 